\title{
FINITE, PRIMITIVE AND EUCLIDEAN SPACES
}

\author{
EFIM KHALIMSKY \\ City University of New York, CSI \\ New York, New York 10040
}

\begin{abstract}
Integer and digital spaces are playing a significant role in digital image processing, computer graphics, computer tomography, robot vision, and many other fields dealing with finitely or countable many objects. It is proven here that every finite $T_{0}$-space is a quotient space of a subspace of some simplex, i.e. of some subspace of a Euclidean space. Thus finite and digital spaces can be considered as abstract simplicial structures of subspaces of Euclidean spaces. Primitive subspaces of finite, digital, and integer spaces are introduced. They prove to be useful in the investigation of connectedness structure, which can be represented as a poset, and also in consideration of the dimension of finite spaces. Essentially $T_{0}$-spaces and finitely connected and primitively path connected spaces are discussed.

Key words: finite topological spaces, digital and integer spaces, digital topology, essentially $T_{0}$-spaces, essentially non- $T_{1}$-spaces, finitely connected spaces, primitive spaces, simplex, poset, linearly ordered set, path connectedness, arcwise connectedness, digital picture processing, abstract complexes.
\end{abstract}

AMS subject classification: 54F05, 54D05, 54F50, $68 \mathrm{U} 05$

This paper continues to build the theory of finite, digital, and integer spaces. See references [7-9] for necessary definitions and terms such as: arc, curve, integer and digital lines, integer spaces, path connectedness, and digital topology, as well as for results which are essential for following the arguments of this paper. For a more complete introduction to the area refer to [6]. Familiarity with topology discussed in references [1-4] is assumed.

\section{INTRODUCTION}

Large-scale systems in fields such as computing, economics, ecology, and engineering are becoming more complex. The complexity of their topology and connectedness structure is also increasing. Nevertheless, they are finite spaces. Graph-theoretical, combinatorial, algebraic and other approaches to such spaces have been used in [9-13].

Digital topology, connectedness, and continuous analogs of digital spaces have received considerable attention in digital image processing, computer vision, computer tomography, and computer graphics [14-17]. The topological terminology has been widely 
used together with the prevailing methodology of discrete mathematics. In order to change this tradition, topological and combinatorial methods have been introduced. This strategy has proven to be productive, obtaining such results as the Digital Jordan Curve Theorem, the Intermediate Value Theorem for digital spaces, the digital closed curve theorem, and digital fundamental groups among others.

It is proven here that finite spaces and digital spaces in particular can be represented as quotient spaces of some subspaces of the Euclidean product spaces. Thus every digital image can be represented as a continuous (quotient) function of a real Euclidean object. The connectedness structure of finite, integer, and some more general spaces is represented as a poset or directed graph. Thus, a functor from the class of finite, integer, and more general topological spaces into the class of posets or directed graphs is built.

\section{BASIC DEFINITIONS AND PROPERTIES}

Definition 1. A space $\mathrm{X}$ is said to be an essentially or inherently non- $T_{1}$-space, iff every connected $T_{1}$-subspace of $\mathrm{X}$ contains at most one point.

Definition 2. $A T_{0^{-s p a c e}} \mathrm{X}$ is said to be an inherently or essentially $T_{0^{-s p a c e}}$, iff every connected $T_{1}$-subspace $\mathrm{Y}$ of $\mathrm{X}$ contains no more than one point.

Definition 3. A space $\mathrm{P}$ is said to be primitive, or a primitive, iff every pair of its points is connected.

Definition 4. A subspace $\mathrm{P}$ of a space $\mathrm{X}$ is said to be primitive, iff every pair of its points is connected, and, for every $\mathrm{x} \notin \mathrm{P}$, there is a point $\mathrm{y} \in \mathrm{P}$ such that the pair $\{\mathrm{x}, \mathrm{y}\}$ is disconnected.

Lemma 1. Every primitive space contains a maximum of one c-point.

Theorem 1. For every pair of points $\{\mathrm{x}, \mathrm{y}\}$ of a primitive space $\mathrm{P}$, there is an open neighborhood of at most one of them, which does not contain the other.

Proof. Assuming the contrary, we get a pair of points $\{x, y\} \subset P$, such that for each one of them there is a neighborhood, which does not contain the other one. Thus each of the points is open in $\{x, y\}$, as a trace of an open subset of $P$. This contradicts the connectedness of the pair of points of a primitive space. 
Corollary 1. For every pair of points of a primitive $T_{0}$-space $\mathrm{P}$, there is an open neighborhood of exactly one of the points which does not contain the other one.

Definition 5. Let $\mathrm{P}$ be a primitive space. If $\mathrm{x}, \mathrm{y} \in \mathrm{P}$ and there is a neighborhood $\mathrm{U}$ of $\mathrm{y}$ such that $\mathrm{x} \notin \mathrm{U}$, then $\mathrm{x}<\mathrm{y}$ or $\mathrm{y}>\mathrm{x}$, and $\operatorname{dim}(\mathrm{y})>\operatorname{dim}(\mathrm{x})$. If every neighborhood of each one of the points $\mathrm{x}$ and $\mathrm{y}$ also contains the other, then $\mathrm{x}$ and $\mathrm{y}$ are noncomparable.

Lemma 2. Let $\mathrm{U}$ and $\mathrm{V}$ be two nonequal open subsets of a primitive set $\mathrm{P}$. Then one of the sets is a subset of the other.

Proof. Let us assume the contrary to the statement of the lemma. Then there is a pair of points $\{x, y\} \subset P$ such that $x \in V, x \notin U, y \in U$, and $y \notin V$. Thus each of the points $x$ and $y$ is open in the pair $\{x, y\}$ as traces of open sets $V$ and $U$ correspondingly. Hence the pair is a disconnected subset of $P$. We get a contradiction of the fact that $P$ is primitive. This proves the lemma.

Theorem 2. Every primitive space $\mathrm{P}$ is an essentially non- $T_{1}$-space.

Proof. Let us assume that there is a connected $\mathrm{T}_{1}$-subspace $\mathrm{Q} \subset \mathrm{P}$, containing at least two points $x$ and $y$. Thus each one of the points $x$ and $y$ is a closed subset of $Q$ and consequently of the pair. That contradicts the connectedness of every pair of points of the primitive space $P$ thus proving the theorem.

Lemma 3. Every essentially non- $T_{1}$-space $\mathrm{X}$ which is a $T_{0^{-}}$-space, is an essentially $T_{0^{-}}$ space. The proof is obvious.

Theorem 3. Every primitive $T_{0}$-space $\mathrm{P}$ is an essentially $T_{0}$-space. The proof follows from the previous lemma and Theorem 2.

Theorem 4. Space $\mathrm{P}$ is a primitive space, if for every pair of points $\{x, y\} \subset \mathrm{P}$ there is a neighborhood of not more than one of them, which does not contain the other.

Proof. Assuming that $\mathrm{P}$ is not a primitive space, we get a disconnected pair $\{x, y\} \subset P$. Thus each one of the sets $\{x\}$ and $\{y\}$ is open in $\{x, y\}$. Hence there are open sets 
$U, V \subset P$ such that $x \in U, y \notin U, y \in V$, and $x \notin V$. This contradicts the conditions of the theorem and shows that $P$ is a primitive space.

Theorem 5. Space $\mathrm{P}$ is a primitive space, iff for every pair $\{\mathrm{x}, \mathrm{y}\} \subset \mathrm{P}$, there is a neighborhood of no more than one of the points, which does not contain the other one. The proof follows from Theorems 3 and 4.

Lemma 4. Let $\mathrm{P}$ be a primitive space. Let the equivalence class $\mathrm{E}(\mathrm{x})$ of every $\mathrm{x} \in \mathrm{P}$ be equal to the set of all points $\mathrm{y} \in \mathrm{P}$, each of which is noncomparable with $\mathrm{x}$. Then the quotient space $\mathrm{Q}$ of the space $\mathrm{P}$ with the equivalence relation described above is a primitive essentially $T_{0}$-space.

Proof. First, we prove that the relation is an equivalence relation. Let $x, y, z \in P$. It is clear that $x$ is equivalent to $x$ and that $y$ is equivalent to $x$ whenever $x$ is equivalent to $y$. Let every open set containing $x$ contain $y$, and every open set containing $y$ contain $z$; then every open set containing $x$ contains $z$. Thus the relation is an equivalence relation.

Let $R$ be the equivalence relation. We prove that $R$ is associated with an order relation on $P$. Let $x R y, t R z$ and $x<t$. The relation $x<t$ means that there is a neighborhood $U(t)$ which does not contain $x$. By the definition of $R, y \notin U$ and $z \in U$. Hence $\mathrm{y}<\mathrm{z}$.

Let $p, q \in Q, x \in p$, and $y \in q$. Because $x$ and $y$ belong to different equivalence classes, they are comparable. Without loss of generality, we can assume that $x<y$. By Theorem $5, y$ is not less than $x$. The same is true for $p$ and $q$. Hence by $5, Q$ is a primitive $\mathrm{T}_{0}$-space.

Theorem 6. Let $\mathrm{P}$ be a primitive space and $\mathrm{x}, \mathrm{y}, \mathrm{z} \in \mathrm{P}$. If $\mathrm{x}<\mathrm{y}$ and $\mathrm{y}<\mathrm{z}$, then $\mathrm{x}<\mathrm{z}$.

Proof. Since $\mathrm{x}<\mathrm{y}$, there is an open set $\mathrm{U}$ such that $\mathrm{y} \in \mathrm{U}$ and $\mathrm{x} \notin \mathrm{U}$. Since $\mathrm{y}<\mathrm{z}$, there is an open set $V$ such that $z \in V$ and $y \notin V$. Hence $y \in U$ and $y \notin V$. By Lemma $2, V \subset U$. Thus $z \in V \subset U$ and $x \notin U$, so $x<z$.

Corollary 2. The set of points of a primitive $T_{0}$-space is linearly ordered by the previously defined relation.

Proof. From Corollary 1 it follows that for every pair of different points $y, x \in P$, either $\mathrm{x}<\mathrm{y}$ or $\mathrm{y}<\mathrm{x}$. From Theorem 6 it follows that $\mathrm{P}$ is linearly ordered. 


\section{THE TOPOLOGY OF PRIMITIVE SPACES}

Theorem 7. Let $\mathrm{P}$ be a primitive space and $\wedge \mathrm{U}(\mathrm{y})$ be the union of all open sets, each one of which does not contain $\mathrm{y}$. Then $\wedge \mathrm{U}(\mathrm{y})=\{\mathrm{x} \mid \mathrm{x}>\mathrm{y}\}$ and it is the greatest among all open subsets of $P$, each one of which does not contain $y$.

Proof. Let $\mathrm{x}>\mathrm{y}$. then, be the definition of ' $>$ ', there is an open set $\mathrm{U}(\mathrm{x})$ such that $y \notin U(x)$. Thus $x \in U(x) \subset \wedge U(y)$, so $\{x \mid x>y\} \subset \wedge U(y)$. Let $x \in \wedge U(y)$. Then there is an open set $V(x)$ such that $x \in V(x)$ and $y \notin V(x)$. Thus $x>y$. Hence ${ }^{\wedge} U(y) \supset\{x \mid x>y\}$. Consequently, $\wedge U(y)=\{x \mid x>y\}$.

Definition 6. Let $\mathrm{P}$ be a set of points of a primitive space $\mathrm{P}$ partially ordered by '<'. A partition of the set $\mathrm{P}$ into two disjoint subsets $\mathrm{A}$ and $\mathrm{B}$ is said to be a cross section of $\mathrm{P}$, iff, for every $\mathrm{x} \in \mathrm{A}$ and $\mathrm{y} \in \mathrm{B}$, either $\mathrm{x}<\mathrm{y}$ or $\mathrm{x}$ and $\mathrm{y}$ are noncomparable.

Lemma 5. Every cross section (A, B) of a primitive space $\mathrm{P}$ is a cross section of one of the following types:

1. A has no max element, B has at least one min;

2. $B$ has no min element, $A$ has at least one max;

3. A has at least one max, $B$ has at least one min, and every max of $A$ is smaller than every min of $\mathrm{B}$;

4. A has at least one max, $B$ has at least one min, and every max of $A$ is not comparable with every min of $\mathrm{B}$;

5. A has no max and $\mathrm{B}$ has no min.

Lemma 6. Let (A, B) be a cross section of a primitive space $\mathrm{P}$. Then the following sets are open:

a) $\mathrm{B}=\{\mathrm{y} \mid \mathrm{y}>\mathrm{x}\}$ is open in cases 2 and 3 ;

b) $\mathrm{B}$ is open in case 5;

c) B is either open or it is not open in case 1;

Proof. Case a) follows from Theorem 7. In case b), let $\mathrm{z} \in \mathrm{B}$. Because there is no $\mathrm{min}$ in $B$, there is a point $t \in B$ such that $t<z$. The set $U=\{y \mid y>t\} \subset B$ is an open set containing $z$. Thus we have that a point belongs to $B$ together with its neighborhood. Therefore $B$ is open. In case 4 of Lemma 5 , assuming that $B$ is open, we get the following: $x \notin B, y \in B$ and so $y>x$. This contradicts the fact that $x$ and $y$ are noncomparable. Thus $B$ is not open. 
Lemma 7. Let $\mathrm{P}$ be a primitive space, $\mathrm{U}$ be an open subset of $\mathrm{P}$, and $\mathrm{x} \in \mathrm{U}$. Then every point $\mathrm{y}$ which is either greater than $\mathrm{x}$ or noncomparable with $\mathrm{x}$ also belongs to $\mathrm{U}$.

Proof. Let $y>x$. Then, by definition of ' $>$ ', there is an open set $V$ such that $y \in V$ and $x \notin V$. By Theorem 5, every open set containing $x$ also contains $y$. Thus $y \in U$.

Lemma 8. Let $\mathrm{P}$ be a poset such that every cross section of it is a cross section of one of the types described in Lemma 5. In order for $\mathrm{P}$ to become a primitive space, it is sufficient to consider as open any family $\mathrm{F}$ of subsets $\mathrm{U}$, each one of which contains any point $\mathrm{X}$ together with all points of $\mathrm{P}$, nonpresiding $\mathbf{x}$.

Proof. For every pair of two different points, $\mathrm{x}$ and $\mathrm{y}$, one of the following relations holds: $1 . x<y ; 2 . y<x ; 3 . x$ and $y$ are noncomparable.

If there is an open set $U \in F$, which contains the smaller point or one of the noncomparable points, then the other point also belongs to $U$. Hence, by Theorem 5 , the poset $P$ with topology $F$ is a primitive space.

Theorem 8. Let $\mathrm{P}$ be a poset with an order relation ' $>$ ', satisfying the following conditions:

1. if $\mathrm{x}$ and $\mathrm{y}$ are noncomparable, and $\mathrm{z}>\mathrm{x}(\mathrm{x}>\mathrm{z})$, then $\mathrm{z}>\mathrm{y}(\mathrm{y}>\mathrm{z})$;

2. if $\mathrm{x}$ and $\mathrm{y}$ are noncomparable, and $\mathrm{y}$ and $\mathrm{z}$ are noncomparable, then $\mathrm{x}$ and $\mathrm{z}$ are noncomparable;

3. if $\mathrm{x}>\mathrm{y}$, then $\mathrm{y} \ngtr \mathrm{x}$;

4. $\mathrm{x}>\mathrm{y}$ and $\mathrm{y}>\mathrm{z}$, then $\mathrm{x}>\mathrm{z}$.

Then in order for $\mathrm{P}$ to become a primitive space, which will induce the same order relation as the poset $\mathrm{P}$, it is necessary and sufficient that the topology $\mathrm{T}$ of $\mathrm{P}$ satisfy the following conditions:

a) $\mathrm{U}(\mathrm{x})=\{\mathrm{y} \mid \mathrm{y}>\mathrm{x}\}$ is open for every $\mathrm{x} \in \mathrm{P}$;

b) $\mathrm{B}$ is open, for every cross section of $\mathrm{P}$ of type 5;

c) $\mathrm{P}$ and the empty set are open;

d) B may or may not be open for any cross section of $\mathrm{P}$ of type 1;

e) every open set $U$ coincides with one of the sets described in a) - d).

Proof. First of all, it is clear that $\mathrm{T}$ is a topology on P. Secondly, every T-open set $\mathrm{U}$ satisfies the conditions of Lemma 8 , and, consequently, $\mathrm{P}$ is a primitive space. 
It is left to prove that the order in the poset $\mathrm{P}$ coincides with the order induced by a topology of the primitive space $P$. Let $x$ and $y$ be elements of the poset $P$ such that $x<y$. Then by a) $U(x)=\{y \mid y>x\}$ is open and $x$ and $y$ are points of the primitive space $P$.

Let $x$ and $y$ be noncomparable elements of the poset $P$. Then, because in case 4 set $\mathrm{B}$ is not $\mathrm{T}$-open, it follows from the condition $\mathrm{e}$ ) that $\mathrm{x}$ and $\mathrm{y}$ are noncomparable as points of a primitive space $P$.

Let $x$ and $y$ be points of the space $P$ such that $x<y$. Then there is a T-open set $U$, such that $y \in U$ and $x \in U$. Set $U$ could be of one of the following types: $a), b$ ), or d). In any of the cases, the open set $U$ is defined so that $x<y$ in the poset for any $y \in U$ and $\mathbf{X E U}$.

Let $\mathrm{x}$ and $\mathrm{y}$ be noncomparable as points of the primitive space $\mathrm{P}$. Then every $\mathrm{T}$ open set $U$ either contains both of the points or does not contain either of them.

Assuming that $x<y$ in the poset, we get from a) a T-open set $U(x)=\{z \mid z>x\}$ with $z \in U(x)$ and $x \notin U(x)$. This contradiction proves that $x$ and $y$ are noncomparable as elements of the poset $P$.

Lemma 9. Let $\mathrm{P}$ be a primitive space and let every point have an immediate predecessor. Then the topology on $\mathrm{P}$ is unique among comparable topologies, and it is defined in the following way: every open set $\mathrm{U}$ is either of the type $\mathrm{U}=\mathrm{U}(\mathrm{y})=\{\mathrm{x} \mid \mathrm{x}>\mathrm{y}\}$ or of the type $\mathrm{U}=\underset{\mathrm{y} \in \mathrm{I} \subset \mathrm{P}}{\cup(\mathrm{U}(\mathrm{y}))}$, where I is a subset of $\mathrm{P}$ such that there is no max element preceding $\mathrm{I}$, or $\mathrm{U}=\mathrm{P}$, or $\mathrm{U}$ is empty. The proof follows from Theorem 8.

Corollary 3. Let $\mathrm{P}$ be a primitive space similar to the set of all integers or to any subset of it. Then there is a unique topology among all comparable topologies on P. The only open sets on $\mathrm{P}$ are the following:

the sets of the type $\{\mathrm{y} \mid \mathrm{y}>\mathrm{x}\}$ for each $\mathrm{x} \in \mathrm{P}$, the empty set, and $\mathrm{P}$.

Lemma 10. Let $\mathrm{x}$ be an element of a primitive space $\mathrm{P}$, which does not have an immediate predecessor. Then there is more than one comparable topology on $\mathrm{P}$. The proof follows from Theorem 8.

Definition 7. A space $\mathrm{X}$ is said to be a primitively path connected space or PPCS, iff for any two points $\mathrm{X}, \mathrm{y} \in \mathrm{X}$ there is a sequence of primitives $\mathrm{P}_{1}, \mathrm{P}_{2}, \ldots, \mathrm{P}_{\mathrm{n}} \subset \mathrm{X}$, such that 
n

$\bigcup_{\mathrm{i}=1}^{\cup} \mathrm{P}_{\mathrm{i}}$ is connected, $\mathrm{x} \in \mathrm{P}_{1}, \mathrm{y} \in \mathrm{P}_{\mathrm{n}}$, and $\mathrm{P}_{\mathrm{i}} \cap \mathrm{P}_{\mathrm{i}-1} \neq \varnothing(\mathrm{i}=2,3, \ldots, \mathrm{n}) . \bigcup_{\mathrm{i}=1}^{\cup} \mathrm{P}_{\mathrm{i}}$ is said to be a primitive path between $\mathrm{x}$ and $\mathrm{y}$.

Definition 8. A space $\mathrm{X}$ is said to be primitively complex, iff it is connected and every point belongs to some primitive subspace of $\mathrm{X}$ containing more than one point.

Definition 9. Let X be a PPCS and all primitive subsets of $\mathrm{X}$ be ordered the way described above. Let $\mathrm{P}_{1}, \mathrm{P}_{2}, \ldots, \mathrm{P}_{\mathrm{n}} \subset \mathrm{X}$ be a primitive path between $\mathrm{X}, \mathrm{y} \in \mathrm{X}$, such that there is $a$ sequence of points $\left\{\mathrm{x}_{\mathrm{i}}\right\}, \mathrm{i}=1,2, \ldots, \mathrm{n}-1$, satisfying the following conditions: $\mathrm{x}_{\mathrm{i}-1} \in \mathrm{P}_{\mathrm{i}} \cap \mathrm{P}_{\mathrm{i}-1}(\mathrm{i}=2, \ldots, \mathrm{n}), \mathrm{x}_{1}<\mathrm{x}_{1}$ in $\mathrm{P}_{1}, \mathrm{x}_{\mathrm{i}-1}<\mathrm{x}_{\mathrm{i}}$ in $\mathrm{P}_{\mathrm{i}}(\mathrm{i}=2, \ldots, \mathrm{n}-1)$, and $\mathrm{x}_{\mathrm{n}-1}<\mathrm{y}$ in $\mathrm{P}_{\mathrm{n}}$. For $\mathrm{x}<\mathrm{y}$, the path $\mathrm{P}_{1}, \mathrm{P}_{2}, \ldots, \mathrm{P}_{\mathrm{n}}$ is called an increasing path from $\mathrm{x}$ to $\mathrm{y}$, and the path $\mathrm{P}_{\mathrm{n}}, \mathrm{P}_{\mathrm{n}-1}, \ldots, \mathrm{P}_{1}$ is called a decreasing path from $\mathrm{y}$ to $\mathrm{x}$. Set $\mathrm{X}$ with the relation ' $<$ ', induced above, is a poset. From now on we shall consider the PPCS together with the relation ' $<$ '.

Lemma 11. Let $\mathrm{P}_{1}, \mathrm{P}_{2}, \ldots, \mathrm{P}_{\mathrm{n}}$ be a monotonic primitive path between $\mathrm{x}$ and $\mathrm{y}$ and let $\mathrm{x}<\mathrm{y}$. Let $\mathrm{P}=\left\{\mathrm{x}, \mathrm{x}_{1}, \mathrm{x}_{2}, \ldots, \mathrm{x}_{\mathrm{n}-1}, \mathrm{y}\right\}$ be the set described in Definition 9. Then $\mathrm{P}$ is $a$ primitive space.

Proof. Let $U$ be an open subset of $X$ containing $x$. Let us assume that $x_{i} \in U$. $x_{i}<x_{i+1} \in P_{i+1}$, by the definition of increasing primitive path. By Lemma $7, x_{i+1} \in U$. Hence, by mathematical induction, every $x_{\mathbf{k}} \in U$ and $y \in U$. Thus every open set containing $x$, contains $y$, whenever $x<y$. Therefore, by Theorem 4 , the space $P$ is a primitive space.

Corollary 4. Let $\mathrm{P}_{1}, \mathrm{P}_{2}, \ldots, \mathrm{P}_{\mathrm{n}}$ be a monotonic primitive path between $\mathrm{x}$ and $\mathrm{y}$. Then the pair $\{\mathrm{x}, \mathrm{y}\}$ is connected and belongs to some primitive subset of $\mathrm{X}$.

Corollary 5. Let there be an order relation between two points $\mathrm{x}$ and $\mathrm{y}$ of space $\mathrm{X}$. Then there is a primitive subspace containing $\mathrm{x}$ and $\mathrm{y}$.

Lemma 12. Let $\mathrm{X}$ be a PPCS. If an open set $\mathrm{U}$ contains a point $\mathrm{X}$, then it also contains every point $\mathrm{y}$ such that either $\mathrm{y}>\mathrm{x}$ or $\mathrm{y}$ is noncomparable with $\mathrm{x}$, and the pair $\{\mathrm{x}, \mathrm{y}\}$ is connected. 
From Corollary 5 it follows that there is a primitive subspace $P \subset X$, containing $\mathrm{X}$ and $y$. From Lemma 7 it follows that $y \in U$.

Lemma 13. Let $\mathrm{X}$ be $a$ PPCS and $\mathrm{x} \neq \mathrm{z}$. If $\mathrm{z} \ngtr \mathrm{x}$ and the pair $\{\mathrm{z}, \mathrm{x}\}$ is not connected, then there is an open set $\mathrm{U}$ such that $\mathrm{x} \in \mathrm{U}$ and $\mathrm{z} \notin \mathrm{U}$.

Proof. If $\mathrm{z} \ngtr \mathrm{x}$, then either $\mathrm{z}<\mathrm{x}$ or $\mathrm{z}$ and $\mathrm{x}$ are noncomparable.

In case 1 , by the definition of the relation ' $<$ ', there is an open set $U$ such that $x \in U$, and $z \notin U$.

In case 2 , the pair $(x, z)$ is nonconnected, so for each of the points there is an open neighborhood which does not contain the other point.

Lemma 14. Let $\mathrm{X}$ be a PPCS which satisfies the conditions of the previous two lemmas and which is such that the intersection of any collection of its open subsets is open. Then for any $\mathrm{x} \in \mathrm{X}$ the set $\mathrm{U}(\mathrm{x})=\{\mathrm{y} \mid \mathrm{y} \geq \mathrm{x}\} \cup \mathrm{E}(\mathrm{x})$ is the minimal open set containing $\mathrm{x}$. $\mathrm{E}$ is a set of all points $\mathrm{y}$ such that $\{\mathrm{x}, \mathrm{y}\}$ is connected and $\mathrm{x}$ and $\mathrm{y}$ are noncomparable.

Proof. The intersection of all open sets containing $\mathbf{x}$ is open by the conditions of this lemma. This intersection is a minimal open set containing $x$. By Lemma 12, it contains all points $y>x$. Every pair $\{x, y\} \subset E$ is connected, so there is a primitive subspace containing $\{x, y\}$. If there were an open set $U$ such that $x \in U$ and $y \notin U$, then $x$ would be greater than $y$. This contradicts the condition that $x$ and $y$ are noncomparable. Thus, $y \in U(x)$. Hence, $E \subset U(x)$. By Lemma 13, $U(x)=\{y \mid y \geq x\} \cup E(x)$.

Corollary 6. Let $\mathrm{X}$ be one of the following spaces: finite $T_{0}$-space, digital space, or integer space. Then $\mathrm{U}(\mathrm{x})=\{\mathrm{y} \mid \mathrm{y} \geq \mathrm{x}\}$ is the minimal open set containing $\mathrm{x}$.

Theorem 9. Let $\mathrm{X}$ be a PPCS. Let $\wedge \mathrm{U}(\mathrm{x})$ be the maximal among all open subsets of $\mathrm{X}$, each one of which does not contain $\mathrm{x}$. Then $\wedge \mathrm{U}(\mathrm{x})=\mathrm{X}-(\{\mathrm{y} \mid \mathrm{y} \leq \mathrm{X}\} \cup \mathrm{E})$. (E is defined in Lemma 14.)

Proof. Let $\{y, x\}$ be connected and $x<y$. Then, by definition of the relation ' $<$ ' on a primitive space, there is an open set $U(y)$ such that $y \in U(y)$ and $x \notin U(y)$. So $\mathrm{y} \in \mathrm{U}(\mathrm{y}) \subset \wedge \mathrm{U}(\mathrm{x})$. 
Let $\{y, x\}$ be disconnected. Then for each of the points, there is an open set which does not contain the other point. Let $U(y)$ be an open set which does not contain $x$. A set $U(x)=\underset{y<x}{\cup} U(y)$ is open, as a union of open sets, and $x \in U(x)$. Thus $y \in U(x) \subset^{\wedge} U(x)$.

Let $y$ and $x$ be noncomparable, and let there be a primitive space containing both of them. Then every open set containing one of them contains the other one. Thus $y \notin \wedge U(x)$.

Let $y<x$ and $U(x)$ be an open set such that $x \notin U(x)$, and let $y \in U(x)$. Then, by Lemma 12, $x \in U(x)$. This contradicts our assumption. Thus we prove that $y \notin U(x)$ whenever $y<x$. Thus $y \star^{\wedge} U(x)$.

Let $y$ and $x$ be noncomparable, and let there be no primitive space containing $\{x, y\}$. Then $\{x, y\}$ is disconnected and for each of the points there is an open set, which does not contain another one. Let $U(y)$ be an open set such that $y \in U(y)$ and $x \notin U(y)$. By the definition of ${ }^{\wedge} U(x), U(y) \subset{ }^{\wedge} U(x)$, and so $y \in U(y) \subset{ }^{\wedge} U(x)$. Thus we prove that, for every $x \in X$, the maximal open set containing $x$ is equal to ${ }^{\wedge} U(x)=X-(\{y \mid y \leq x\} \cup E)$.

Theorem 10. Let $\mathrm{X}$ be a PPCS satisfying the conditions of Lemma 14; then for any $\mathrm{x} \in \mathrm{X}$ the set ${ }^{\wedge} \mathrm{U}(\mathrm{x})=\{\mathrm{y} \mid \mathrm{y}>\mathrm{x}\}$ is open.

Proof. Let $\mathrm{y}>\mathrm{x}$; then there is a primitive space $\mathrm{P} \subset \mathrm{X}$ such that $\mathrm{x}, \mathrm{y} \in \mathrm{P} \subset \mathrm{X}$. There is an open subset $V \subset P$ such that $y \in V$ and $x \notin V$. Open in $P$, set $V$ is a trace of some set $U(y) \subset X$, open in $X$, i.e. $V=U(y) \cap P . x \in U$ and $y \in U$. Thus, for every $y>x$, there is an open set $U(y)$, containing $y \cdot{ }^{\wedge} U(x) \subset \underset{y>x}{\cup U(y)}$. Because $x \notin \underset{y>x}{\cup} U(y)$, neither the point $z<x$, nor the point $z$ noncomparable and connected with $x$, belongs to $U(y)$. If $z$ $y>x$

is noncomparable with $x$ and if there is no primitive space containing $x$ and $z$, then $\mathrm{z} \notin \cup \mathrm{U}(\mathrm{y})$. $y>x$

Corollary 7. The sets of types: $\mathrm{V}(\mathrm{x})=\{\mathrm{y} \mid \mathrm{y}>\mathrm{x}\}, \mathrm{U}(\mathrm{x})=\mathrm{X}-\{\mathrm{y} \mid \mathrm{y} \leq \mathrm{x}\}$, and ${ }^{\wedge} \mathrm{U}(\mathrm{x})=$ $\{\mathrm{y} \mid \mathrm{y} \geq \mathrm{x}\}$, are open in finite $T_{0^{-s p a c e s}}$ and digital and integer spaces. The proof follows from Lemma 14 and Theorems 9 and 10.

Theorem 11. Let X be a PPCS such that an intersection of any collection of its open subsets is open. Then there is a unique $T_{0}$-topology among all comparable topologies, producing the same set of connected subsets of $\mathrm{X}$. The open sets are of types: 
$\wedge U(x)=\{y \mid y>x\}, V(x)=\{y \mid y \geq x\} \cup E$, and $U(x)=X-\{y \mid y \leq x\}-E$, where $x$, $y \in X$. The proof follows from Lemma 14 and Theorems 9 and 10.

Corollary 8. Finite $T_{0}$-spaces and digital and integer spaces each have a unique topology among all comparable $T_{0}$-topologies, producing the same set of connected subspaces. The topology of these spaces is described above.

Definition 10. Let $\mathrm{X}$ be a primitively path connected $T_{0}$-space such that every primitive subset of $\mathrm{X}$ has the first element, and for every $\mathrm{y} \in \mathrm{X}$, whenever there is an element $\mathrm{X}<\mathrm{y}$, there is an immediate predecessor $\mathrm{z}<\mathrm{y}$ such that $(\mathrm{z}, \mathrm{y})=\varnothing$. We assign to the first element $\mathrm{x}$ of every primitive set $\mathrm{P} \operatorname{dim}(\mathrm{x}, \mathrm{P})=0$ if $\mathrm{x}$ is a c-point and $\operatorname{dim}(\mathrm{x}, \mathrm{P})=1$ in all other cases. By induction, $\operatorname{dim}(\mathrm{x}, \mathrm{P})=\mathrm{k}$ if the $\operatorname{dim}(\mathrm{y}, \mathrm{P})=\mathrm{k}-1$, where $\mathrm{y}$ is the immediate predecessor of $\mathrm{x}$.

Let $\mathrm{x} \in \mathrm{P}_{1} \cap \mathrm{P}_{2} \cap \ldots \cap \mathrm{P}_{\mathrm{k}}$ and $\mathrm{d}_{1}, \mathrm{~d}_{2}, \ldots, \mathrm{d}_{\mathrm{k}}$ be the corresponding dimensions of $\mathrm{x}$ in $\mathrm{P}_{1}, \mathrm{P}_{2}, \ldots, \mathrm{P}_{\mathrm{k}}$. Let $\mathrm{D}(\mathrm{x})=\max \left(\mathrm{d}_{1}, \mathrm{~d}_{2}, \ldots, \mathrm{d}_{\mathrm{k}}\right)$. By induction, we redefine $\operatorname{dim}\left(\mathrm{y}, \mathrm{P}_{\mathrm{i}}\right)$ for every $\mathrm{y} \geq \mathrm{x}$ (shift the dim). Continuing this process, it is possible for us to assign to each point $\mathrm{x} \in \mathrm{X}$ an ordinal number $\mathrm{D}(\mathrm{x})$ such that $\mathrm{D}(\mathrm{x})<\mathrm{D}(\mathrm{y})$, whenever $\mathrm{x}<\mathrm{y}$. We call point $\mathrm{x}$ an $i$-point if $\mathrm{D}(\mathrm{x})=\mathrm{i}$. For simplicity, we restrict in some cases our consideration to finite $T_{0}$-spaces.

Definition 11. Let the pair $\{x, y\}$ be connected and $D(x)=i, D(y)=j$ and $j<i$. Then point $\mathrm{y}$ is called a $j$-face of $i$-point $\mathrm{x}$.

\section{FINITE AND FINITELY CONNECTED SPACES}

Theorem 12. Every finite space $\mathrm{X}$ is an essentially non- $T_{1}$-space.

Proof. Let us assume that there is a finite space $\mathrm{X}$ which has a connected $\mathrm{T}_{1}$-subspace $\mathrm{Y}$, containing more than one point. $\mathrm{Y}$ is finite and every one-point subset of $\mathrm{Y}$ is closed. The set $Z=Y-\{y\}$ is a closed subset of $Y$ as a finite union of closed sets. Thus $Y$ is represented as a union of two disjoint closed subsets. A contradiction to the connectedness of $Y$ proves the theorem. 
Theorem 13. Every finite $T_{0}$-space is an essentially $T_{0}$-space. The proof follows from Theorem 12 and the fact that every $T_{0}$-space is an essentially $T_{0}$-space, whenever it is an essentially non- $\mathrm{T}_{1}$-space.

Lemma 15. Every PPCS is path connected. The proof is obvious.

Theorem 14. Space $\mathrm{X}$ is a PPCS if, for every pair of points $\{\mathrm{x}, \mathrm{y}\} \subset \mathrm{X}$, there is a finite arc connecting them.

Proof. Every finite arc $\mathrm{C}$ is either a $\mathrm{T}_{0}$-arc or the antidiscrete two-point set, unless it is not $a$ one-point set. In any case, $C$ is a finite sequence of points, $x=c_{1}, c_{2}, \ldots, c_{n}=y$, such that every pair $\left\{c_{i}, c_{i+1}\right\}$ is connected $(i=1, \ldots, n-1)$. Thus every pair $\left\{c_{i}, c_{i+1}\right\}$ $(i=1,2, \ldots, n-1)$ belongs to some primitive subset $P$ of $X$. Hence $X$ is PPCS.

Definition 12. Space $\mathrm{X}$ is said to be finitely connected, iff for every pair of its points $\{\mathrm{x}, \mathrm{y}\}$ there is a finite arc, $\mathrm{C} \subset \mathrm{X}$, connecting them.

Hence we proved the following.

Lemma 16. Every finitely connected space $\mathrm{X}$ is a PPCS.

Remark 1. A finitely connected space is not finite in general; it can have any cardinality.

Lemma 17. Every PPCS is finitely connected. The proof follows immediately from the definitions.

Example. Not every path connected essentially $T_{0}$-space $\mathrm{X}$ is arcwise connected.

Let $X=I \cup\{x, y\}$, where $I$ is an integer line with its order and topology. Every set of types $[n, \infty) \cup\{x\}$ and $[n, \infty) \cup\{y\}$ is open in $X$, whenever $\{n\}$ is open in I. For every pair of open subsets $\{n\}$ and $U$ of $I$, the sets of types: $U, U \cup[n, \infty) \cup\{x\}$, $U \cup[n, \infty) \cup\{y\}, U \cup[n, \infty) \cup\{x\} \cup\{y\}, X$ and the empty set are open subsets of $\mathrm{X}$.

It is clear that $X$ is a path connected essentially $T_{0}$-space, which is not arcwise connected. There is no arc in $X$ connecting points $\mathrm{X}$ and $\mathrm{y}$. Thus $\mathrm{X}$ is not finitely connected. 


\section{FINITE, DIGITAL, AND EUCLIDEAN SPACES}

Lemma 18. The number $\mathrm{N}(\mathrm{Sn}, \mathrm{k})$ of $\mathrm{k}$-faces of an $\mathrm{n}$-simplex $\mathrm{Sn}$ is equal to $\left(\begin{array}{c}\mathrm{k}+1 \\ \mathrm{n}+1\end{array}\right)$, and it approaches infinity, when $\mathrm{n} \rightarrow \infty$. The proof is obvious.

Lemma 19. The number $\mathrm{M}(\mathrm{N}, \mathrm{j}, \mathrm{i})$ of $\mathrm{J}$-faces of an $\mathrm{N}$-simplex, each one of which has common i-face with other $\mathrm{j}$-faces, is equal to $\left(\begin{array}{c}\mathrm{j}-\mathrm{i} \\ \mathrm{N}-\mathrm{i}\end{array}\right)$, and it approaches infinity, when $\mathrm{N}$ approaches infinity. The proof is obvious.

For $i=1,2, \ldots, k$, where $k$ is one greater than the highest dimension of a point, we are checking each $i$-point of $X$. If, for some $i$-point $x$ the number $N(i, j)$ of $j$-points $(j-$ faces) connected with $x$ in $X$ is greater than the number $N(S i, j)$ of $j$-faces of $S i$, i.e. $N(i, j)$ $>\mathrm{N}(\mathrm{Si}, \mathrm{j})$, then we increase the $\mathrm{D}$ of this $\mathrm{i}$-point and the $\mathrm{D}$ of all the points which are greater than $x$. We continue increasing $D$ until $N(S i, j)$ becomes greater than $N(i, j)$. By Lemma 18 , it is possible to achieve the wanted result.

Let $\mathrm{N}$ be the highest $\mathrm{D}$ of a point of $\mathrm{X}$ plus 1 , after the end of the previous sequence of checkings. For $I=0,1, \ldots, N-1$, we shall compare for each $i$-point $x$, the number of $j$-points $N(x, j)$, for which $x$ is an i-face, with the number $M(N, j, i)$. If $M(N, j, i)>$ $N(x, j)$, then we can choose $N$ such that $M(N, j, i)>N(x, j)$. This is possible to do, by Lemma 19.

Remark 2. In previous procedures, we added to $\mathrm{X}$ one more point $\mathrm{x}_{0}$, such that for any $\mathrm{x} \in \mathrm{X}$, the pair $\left\{\mathrm{x}_{0}, \mathrm{x}\right\}$ is connected. Thus $\mathrm{x}_{0}$ belongs to every primitive set, $\mathrm{x}_{0}$ is the greatest element of $\mathrm{X}$, and every point $\mathrm{x}$ is a face of $\mathrm{x}_{0}$.

Below we consider only open simplexes in Euclidean spaces, without points belonging to their faces.

Let the simplex $S N$ correspond to $x_{0}$. We assign to each $i$-point of $X$ an $i$-face of $\mathrm{SN}$ in such a way that $\mathrm{Si}$ is an $\mathrm{i}$-face of $\mathrm{Sj}$, if the corresponding $\mathrm{i}$-point is an $\mathrm{i}$-face of a corresponding $\mathrm{j}$-point. This correspondence relation is a monomorphism, which preserves the connectedness relation. Let us take a subset of a closed simplex SN, containing only its faces, which correspond to the points of $X$. This subspace of SN we call SN(X). The quotient space of $S N(X)$ is obtained from it by representation of each face of $S N$, belonging to $\operatorname{SN}(X)$, as one point. The obtained quotient space $\operatorname{ASN}(X)$ we call an abstract complex, corresponding to the union of open simplexes SN(X). The abstract complex ASN $(\mathrm{X})$ is homeomorphic to $\mathrm{X}$, by Corollary 8 . 
By induction it is easy to prove that $\mathrm{SN}(\mathrm{X})$ can be built in finitely many steps for the finite $T_{0}$-space $X$. We start from 0-points. They can take arbitrary vertex positions in SN. Simplexes are used because all their $\mathrm{i}$-faces have the same local connectedness structure. Next, we consider 1-points of $X$. Let us assume that we already positioned all $i-$ points for $i=1,2, \ldots, k-1$ and some $k$-points. Now we try to find a place for a k-point $x$ in $\mathrm{SN}(\mathrm{X})$. When it is impossible, we increase the dimension of $\mathrm{X}$ and of all the points of $\mathrm{X}$ which are greater than $x$, increasing them on the same value by shifting them. The value of $\mathrm{N}$, i.e., the dimension of SN, will increase correspondingly. By Lemma 18, it is always possible to satisfy any connectedness relation. We have to take care only about faces, because points of higher dimension have not been positioned. Points of the same dimension for $\mathrm{T}_{0}$-spaces are disconnected. We can also give geometrical proof. If the $\mathrm{i}$-point $\mathrm{y}$ is less than the $\mathrm{k}$-point $\mathrm{x}$, then the simplex $\operatorname{Si}(\mathrm{y})$, corresponding to $\mathrm{y}$, is a face of a simplex $\mathrm{Sk}(\mathrm{x})$, corresponding to $\mathrm{x}$. Let $\mathrm{Vi}(\mathrm{y})$ and $\mathrm{Vk}(\mathrm{x})$ be respectively the sets of vertices of $\operatorname{Si}(y)$ and $\operatorname{Sk}(x) . V i(y) \subset V k(x)$, because $\operatorname{Si}(y)$ is a face of $\operatorname{Sk}(x)$. If $z<y$, then $z<x$, and so $z$ is a face of $x$. Let $U$ be a union of vertices of all faces of $x$. If there is a $k$-simplex $S k$ in $S N(X)$, for which $U$ is a subset of its vertices, and if no one other vertex of simplexes, which have been positioned before, belongs to Sk, then Sk can represent $\mathrm{X}$. Otherwise, if there are $n$ points in $U$ and if $m$ points satisfying the previously-stated condition can be found in $\mathrm{SN}$, then we increase the dimension of $\mathrm{SN}$ and of each point $z>x$ by $j=k-m-n+1$. Let us take $j$ points of the new SN, which do not belong to $\mathrm{SN}(\mathrm{X})$. We get $\mathrm{k}+1$ vertices of a new simplex, which we call $\mathrm{Sk}(\mathrm{x})$. We add this $k$-simplex to $\operatorname{SN}(\mathrm{X})$. It is clear that $\mathrm{Sk}(\mathrm{x})$ has the same connectedness relations with the simplexes of $\mathrm{SN}(\mathrm{X})$ of lower dimensions, as $\mathrm{x}$ does with corresponding points of $\mathrm{X}$. By induction, this process can be repeated any finite number of times. Thus, we construct $\mathrm{SN}(\mathrm{X}) \subset \mathrm{SN}$, repeating the process $\mathrm{n}$ times, where $\mathrm{n}$ is the number of points of $\mathrm{X}$.

We get the following:

Theorem 15. Every finite connected $T_{0^{-}}$space $\mathrm{X}$ is a quotient space of a subspace $\mathrm{SN}(\mathrm{X})$ of some simplex SN. SN(X) is a union of some faces of simplex SN. There is a one-toone correspondence between $\mathrm{X}$ and the faces of $\mathrm{SN}$, which belong to $\mathrm{SN}(\mathrm{X})$. $\mathrm{X}$ is a quotient space of $\mathrm{SN}(\mathrm{X})$, obtained by replacing each simplex of $\mathrm{SN}(\mathrm{X})$ with one point. The connectedness relation between simplexes of $\mathrm{SN}(\mathrm{X})$ is the same as the connectedness relation between corresponding points of $\mathrm{X}$.

Theorem 16. Every finite $T_{0^{-s p a c e}} \mathrm{X}$ is a quotient space of some subspace $\mathrm{SN}(\mathrm{X})$ of Euclidean space $\mathrm{E}(\mathrm{N})$, which can be represented as a finite disjoint union of open 
simplexes $\mathrm{ASN}(\mathrm{X})$. The quotient function maps every simplex of $\mathrm{SN}(\mathrm{X})$ into a corresponding point of $\mathrm{X}$ and preserves the connectedness relation between simplexes in $\mathrm{SN}(\mathrm{X})$.

Definition 13. Let $\mathrm{P}$ be a poset. Let any two distinct elements $\mathrm{x}$ and $\mathrm{y}$ of $\mathrm{P}$ satisfy exactly one of the following relations:

a) $\mathrm{x}>\mathrm{y}$ (order relation);

b) $\mathrm{x} \sim \mathrm{y}$ (equivalence relation);

c) $\mathrm{xly}$ (noncomparability or separation relation).

The poset $\mathrm{P}$ is said to be a poset enriched with the equivalence relation ' $\sim$ ', iff for any $\mathrm{a}, \mathrm{b}, \mathrm{c}, \mathrm{d} \in \mathrm{P}$ the following conditions are satisfied:

1) if $\mathrm{a}>\mathrm{b}$, then $\mathrm{b}>\mathrm{a}$;

2) if $\mathrm{a}>\mathrm{b}$ and $\mathrm{b}>\mathrm{c}$, then $\mathrm{a}>\mathrm{c}$;

3) if $\mathrm{a} \sim \mathrm{b}, \mathrm{c} \sim \mathrm{d}$ and $\mathrm{a}>\mathrm{c}$, then $\mathrm{b}>\mathrm{d}$;

4) if $\mathrm{a} \sim \mathrm{b}, \mathrm{c} \sim \mathrm{d}$ and $\mathrm{akc}$, then $\mathrm{bld}$.

We say that $\mathrm{b}<\mathrm{a}$ iff $\mathrm{a}>\mathrm{b}$.

Lemma 20. Let $\mathrm{X}$ be an essentially non- $\mathrm{T}_{1}$-space, and $\mathrm{x}$ and $\mathrm{y}$ be two arbitrary points of $X$. Then one of the following holds for every pair of points $\{x, y\}$ of $X$ :

a) $\mathrm{x}>\mathrm{y}(\mathrm{y}>\mathrm{x})$, if $\{\mathrm{x}, \mathrm{y}\}$ is connected and there is an open neighborhood of $\mathrm{y}(\mathrm{x})$ which does not contain $\mathrm{x}(\mathrm{y})$;

b) $\mathrm{x} \sim \mathrm{y}$, if $\{\mathrm{x}, \mathrm{y}\}$ is connected and every open neighborhood of one of the points contains the other one as well;

c) $\mathrm{x} \backslash \mathrm{y}$ if $\{\mathrm{x}, \mathrm{y}\}$ is disconnected.

$A$ poset $\mathrm{P}$ of points of $\mathrm{X}$ is a poset, enriched with the equivalence relation ' $\sim$ '.

The proof follows immediately from previous lemmas and theorems.

Corollary 9. A poset $\mathrm{P}$ is a non-enriched poset if $\mathrm{X}$ is an essentially $T_{0^{-s p a c e . ~}}$

Definition 14. Let $\mathrm{V}$ be a subspace of the space $\mathrm{X}$. A subset $\mathrm{S}(\mathrm{V})$ of the space $\mathrm{X}$ is said to be a surrounding of $\mathrm{V}$ in $\mathrm{X}$, iff every connected set $\mathrm{U}$ of $\mathrm{X}$, satisfying the inequality $\mathrm{U} \cap \mathrm{V} \neq \varnothing \neq \mathrm{U} \cap(\mathrm{X}-\mathrm{V})$, also satisfies the inequality $\mathrm{S}(\mathrm{V}) \cap \mathrm{U}-\mathrm{V} \neq \varnothing$.

The notion of surrounding is very important. It plays the same role for $\mathrm{T}_{0}$-spaces and for spaces without any separation axiom as neighborhood does for Hausdorff spaces. 
Definition 15. A subset $\mathrm{S}(\mathrm{V})$ of the space $\mathrm{X}$ is said to be a direct surrounding of $\mathrm{V} \subset \mathrm{X}$ in $\mathrm{X}$, iff $\mathrm{S}(\mathrm{V})$ is a surrounding of $\mathrm{V}$, and for every point $\mathrm{x} \in \mathrm{S}(\mathrm{V})-\mathrm{V}$ there is a point $\mathrm{y} \in \mathrm{V}$ such that $\{\mathrm{x}, \mathrm{y}\}$ is connected.

It is clear that direct surrounding is the smallest surrounding of a set in space.

Theorem 17. Let $\mathrm{X}$ be a connected $T_{0}$-space. Then for every $\mathrm{x} \in \mathrm{X}$ one of the following statements holds:

1. there is a direct surrounding $\mathrm{S}(\mathrm{x})$ of $\mathrm{x}$ in $\mathrm{X}$;

2. there is a subset $\mathrm{V}$ of $\mathrm{X}-\{\mathrm{x}\}$ such that $\mathrm{x} \in \mathrm{Cl}(\mathrm{V}), \mathrm{X}$ is a c-point of $\mathrm{X}$, and $\{\mathrm{x}, \mathrm{y}\}$ is disconnected for every $\mathrm{y} \in \mathrm{V}$. Every neighborhood of $\mathrm{x}$ contains infinitely many points of $\mathrm{V}$.

Proof. Let $\mathrm{X}$ be a $\mathrm{T}_{0}$-space and $\mathrm{x} \in \mathrm{X}$. Let $\mathrm{V}(\mathrm{x})$ be an intersection of all open subsets of $\mathrm{X}$ containing $\mathrm{x}$. There are two possible cases:

1. $\mathrm{V}(\mathrm{x})=\{\mathrm{x}\}$;

2. $V(x)$ contains some points different from $x$.

Let $V(x)=\{x\}$ and $x$ be a c-point. By definition of $V(x)$, for any $y$ there is an open neighborhood $U(x)$ such that $y \notin V(x)$. Thus $\{x\}$ is a clopen subset of $\{x, y\}$ and the pair $\{x, y\}$ is disconnected.

Every neighborhood of $x$ contains infinitely many points. Assuming the contrary, we get a neighborhood $U$ of $x$, which contains finitely many points. For any $y$, different from $x$, there is an open set $W$ such that $x \in W$ and $y \notin W$. Thus $\{x\}$ is open as the intersection of finitely many open sets. Hence, $\{x\}$ is a clopen subset of $X$. The contradiction with the fact that $X$ is connected shows that every neighborhood of $X$ contains infinitely many points.

Let $V(x)=\{x\}$ and $x$ not be a c-point. Let $y \in C l(x)$. Then $\{x, y\}$ is connected; otherwise open sets $U(x)$ and $U(y)$ would be separating $x$ from $y$, i.e., $y \notin U(x)$ and $x \notin U(y)$. $y \in C l(x)$ implies that $x \in U(y)$. Thus $\{x, y\}$ is connected. If $z \notin C l(x)$, then $\{x, z\}$ is disconnected. By definition of $\mathrm{Cl}(\mathrm{x})$, there is a $\mathrm{U}(\mathrm{z})$ such that $\mathrm{x} \in \mathrm{U}(\mathrm{z})$. There is a $\mathrm{U}(\mathrm{x})$ such that $z \notin U(x)$, because $\cap U(x)=\{x\}$.

Let $V(x) \neq\{x\}$ and $y \in V(x)$. Because $V(x)$ is the intersection of all open subsets of $X$ containing $x$, every open neighborhood $U(x)$ of $x$ contains $y$. Thus $\{x, y\}$ is connected. 
Definition 16. A continuous map $\mathrm{f}: \mathrm{Y} \rightarrow \mathrm{X}$ is said to be a connectedness-preserving map, iff the coimage of every connected subset of $\mathrm{X}$ is a connected subset of $\mathrm{Y}$.

Theorem 18. For every $T_{0}$-space $\mathrm{X}$ there is an effective way of constructing a corresponding $T_{2}$-space (Hausdorff space) $\mathrm{H}(\mathrm{X})$, such that there is a connectednesspreserving quotient map $\mathrm{f}: \mathrm{H}(\mathrm{X}) \rightarrow \mathrm{X}$.

Proof. We consider all c-points of $\mathrm{X}$ as points of $\mathrm{H}(\mathrm{X})$, For every non-c-point $\mathrm{x} \in \mathrm{X}$, by the previous theorem, there is a direct surrounding $S(x)$. Let $y \in S(x)$. We connect the points $x$ and $y$ with the interval $(0,1)$ of the real line.

If $x$ is an o-point, then for every $y \in S(x)$ we connect points $x$ and $y$ with some interval homeomorphic to the interval $(0,1)$ of the real line:

a) the interval $(0,1)$ is associated with $x$, if $y$ is a c-point;

b) the interval $(0,1 / 2)$ is associated with $x$, if $y$ is an $n$-point.

The same is done with every o-point $x$.

If $x$ is an $n$-point, then for every $y \in S(x)$ we connect $x$ and $y$ with the interval $(0,1)$, if they are not connected yet:

a) the interval $(0,1)$ is associated with $x$, if $y$ is a c-point;

b) the interval $[1 / 2,1)$ is associated with $x$, if $y$ is an o-point;

c) one of the intervals $(0,1 / 2),(0,1 / 2],(1 / 2,1)$, or $[1 / 2,1)$ is associated with $x$, if $y$ is an n-point, and the other half of the interval $(0,1)$ is associated with $y$.

Every point $x \in X$ is associated with $x$ together with the union of all intervals associated with this point. The topology on every copy of $(0,1)$ is induced by the topology of the real line. The points $x$ and $y$ replace the endpoints of $(0,1)$. The set that we get this way is denoted as $H(X)$. The topology on the space is natural, and the quotient function $f: H(X) \rightarrow X$ maps the point $x$ together with the union of all associated intervals from $H(X)$ into the point $X$ of $X$. It is obvious that $f$ is a connectedness function.

Definition 17. Let $\mathrm{X}$ be a topological union of some family of mutually disjoint open convex polytopes. Let $\mathrm{K}(\mathrm{X})$ be a quotient space of $\mathrm{X}$ such that points of $\mathrm{K}(\mathrm{X})$ are polytopes of $\mathrm{X}$. Then $\mathrm{K}(\mathrm{X})$ is said to be a topological complex of $\mathrm{X}$.

Lemma 21. Let $\mathrm{X}$ be a topological union of some family of mutually disjoint open convex polytopes and let $\mathrm{f}: \mathrm{X} \rightarrow \mathrm{K}(\mathrm{X})$ be a quotient map described in Definition 17. Then $\mathrm{f}(\mathrm{x})$ is a connectedness-preserving map. The proof follows from definitions of $f(x)$ and polytopes. 
Theorem 19. Let $\mathrm{X}$ be a finite $T_{0}$-space. There exists a simplex $\overline{\mathrm{Sn}}$ such that $\mathrm{X}$ can be embedded into $\mathrm{K}(\overline{\mathrm{Sn}})$. The proof follows from Theorem 15.

Theorem 20. Let $\overline{\mathrm{Sn}}$ be a closed n-simplex and $\mathrm{e}_{\mathrm{n}+1}$ be a closed $(n+1)$-cube. Then $\mathrm{K}(\overline{\mathrm{Sn}})$ can be embedded into $\mathrm{K}\left(\mathrm{e}_{\mathrm{n}+1}\right)$.

Proof. $\mathrm{K}\left(\mathrm{e}_{\mathrm{n}+1}\right)$ is a topological product of $(\mathrm{n}+1)$ copies of a segment $[1,3]$ of the integer line. Let $\left\{a_{1}, a_{2}, \ldots, a_{n+1}\right\}$ be the set of vertices of the simplex. We assign to a vertex $a_{i}$ the point of $\mathrm{K}\left(\mathrm{e}_{\mathrm{n}+1}\right)$ such that the $\mathrm{i}$-th coordinate of the point is equal to 3 and all other coordinates are equal to 1 .

Let a be a point of $\mathrm{K}(\overline{\mathrm{Sn}})$, which is an image of an i-face of $\overline{\mathrm{Sn}}$, associated with vertices $\left(a_{i_{1}}, a_{i_{2}}, \ldots, a_{i_{k}}\right)$ of $S n$. We assign to a $K(\overrightarrow{S n})$ the point $\left(b_{1}, b_{2}, \ldots\right.$, $\left.b_{n+1}\right) \in K\left(e_{n+1}\right)$, where $b_{i_{1}}=b_{i_{2}}=\ldots=b_{i_{k}}=2$ and all other coordinates are equal to 1 .

It is obvious that the only c-points in the image of $\mathrm{K}(\overline{\mathrm{Sn}})$ in $\mathrm{K}\left(\mathrm{e}_{\mathrm{n}+1}\right)$ are the images of vertices and the only o-point in $\mathrm{K}\left(e_{\mathrm{n}+1}\right)$ is $(2,2, \ldots, 2)$, which is an image of $\mathrm{K}(\overline{\mathrm{Sn}})$. It is clear that the map of $\mathrm{K}(\overline{\mathrm{Sn}})$ into $\mathrm{K}\left(\mathrm{e}_{\mathrm{n}+1}\right)$ is a connectedness-preserving map.

\section{URYSON'S THEOREM FOR INTEGER PRODUCT SPACES}

Theorem 21. Every finite $T_{0}$-space can be embedded into some integer product space. The proof follows from Theorems 19 and 20.

\section{REFERENCES}

[1] Bourbaki, N., Topologie General, Herman, Paris, [1953].

[2] Greenberg, M., Lectures on Algebraic Topology, Benjamin, New York, [1967].

[3] Hurewicz, W. and H. Wallman, Dimension Theory, Princeton U. Press, Princeton, NJ, [1941].

[4] Kelley, J., General Topology, Van Nostrand, New York, [1955], Springer-Verlag, [1975].

[5] Khalimsky, E., On Topologies of Generalized Segments, Sov. Math. Docl. 10(2), 351-353, [1969]. 
[6] _ Ordered Topological Spaces and Their Applications, Naukova Dumka Press, Kiev, [1977].

[7] _ Pattern Analysis of N-dimensional Digital Images, Proc. IEEE Conf.: Systems, Man, and Cybernatics, Atlanta, GA, 1559-1563, [1986].

[8] Topological Structures in Computer Science, JAMS Vol 1, No. 1, 26-40, [1987].

[9] Motion, Deformation and Homotopy in Finite Spaces, Proc. IEEE Conf.: Syst., Man, Cyb., 227-234, [1987].

[10] Kong, T., A. Rosenfeld, Digital Topology, Report Car TR 229, Coll. Park, MD [1986].

[11] Kong, T., and A. Roscoe, A Theory of Binary Digital Pictures, Comp. Vision, 32, 22-24, [1985].

[12] Minsky, M. and S. Papert, Perceptron: An Intro. to Comp. Geom., MIT Press, Cambridge, MA, [1969].

[13] Mylopulos, J. and T. Pavlidis, On the Topological Properties of Quantized Spaces., J. ACM 18(2), 239-246 [1971].

[14] Rosenfeld, A., Digital Topology, AMS Monthly, 86(8): 621-630 [1979].

[15] Connectivity in Digital Pictures, J. Assoc. Comp. Mach. 17, 146-160, [1970]. Arcs and Curves in Digital Pictures, J. Assoc. of Comp. Mach. 20, 81-87, [1987]. 
196 JOURNAL OF APPLIED MATHEMATICS AND SIMULATION Volume 1, Number 3, 1988

[18] Udura, J. and S. Srihary, Boundary Detection in Multidimensions, IEEE Trans. Pattern Anal., 4(1), 41-62, [1982]. 


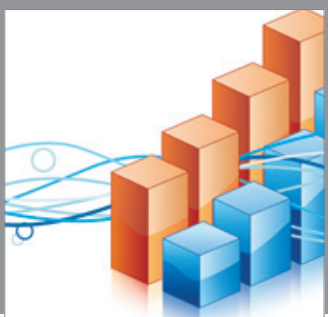

Advances in

Operations Research

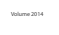

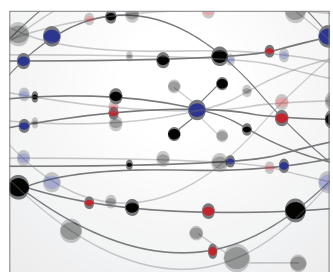

\section{The Scientific} World Journal
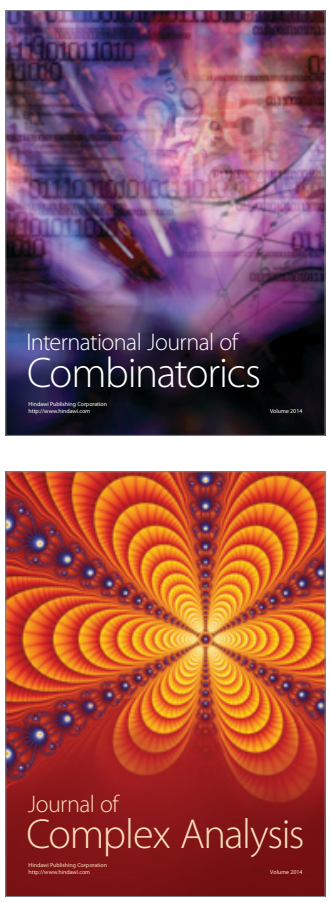

International Journal of

Mathematics and

Mathematical

Sciences
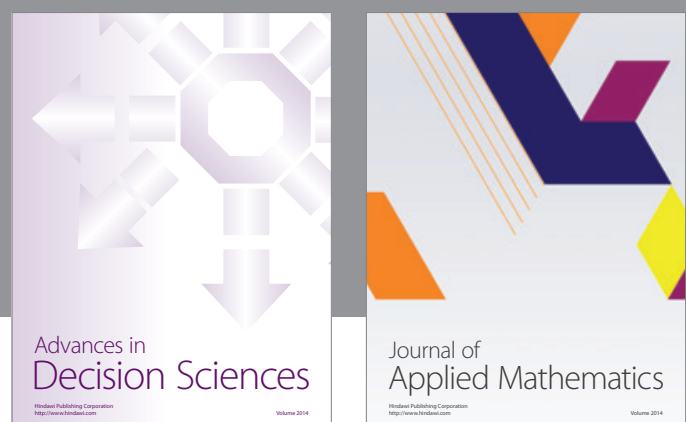

Journal of

Applied Mathematics
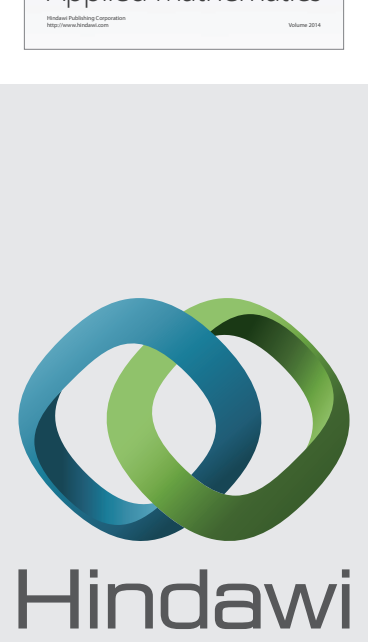

Submit your manuscripts at http://www.hindawi.com
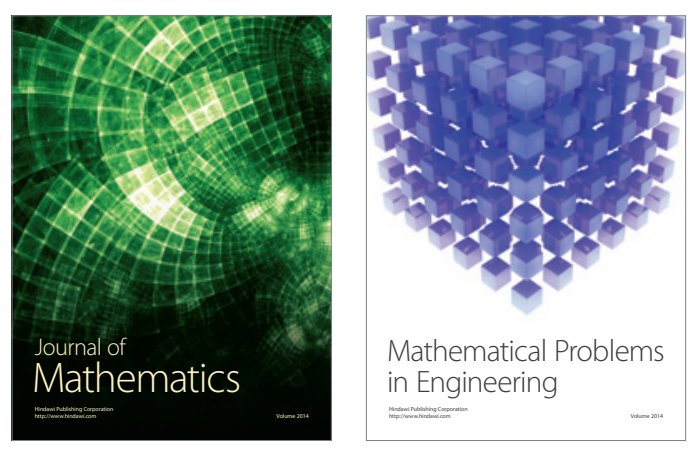

Mathematical Problems in Engineering
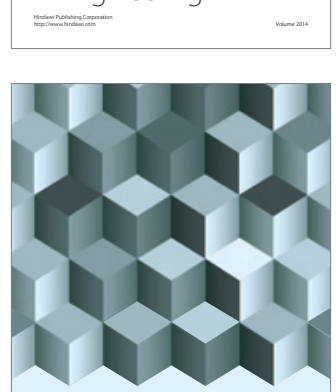

Journal of

Function Spaces
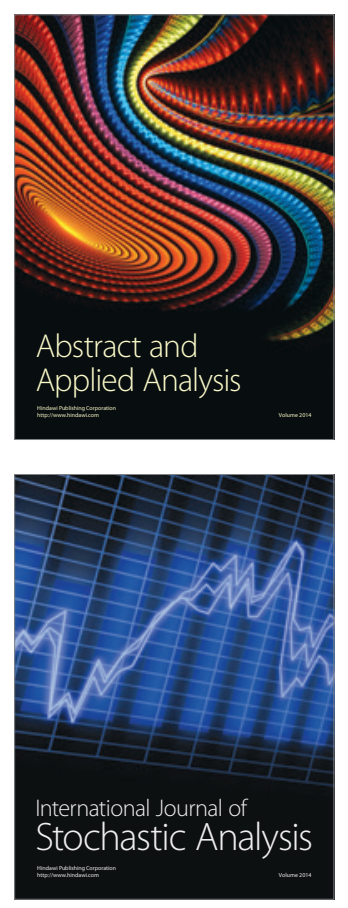

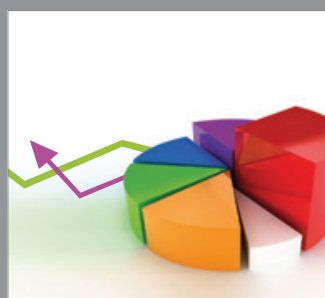

ournal of

Probability and Statistics

Promensencen
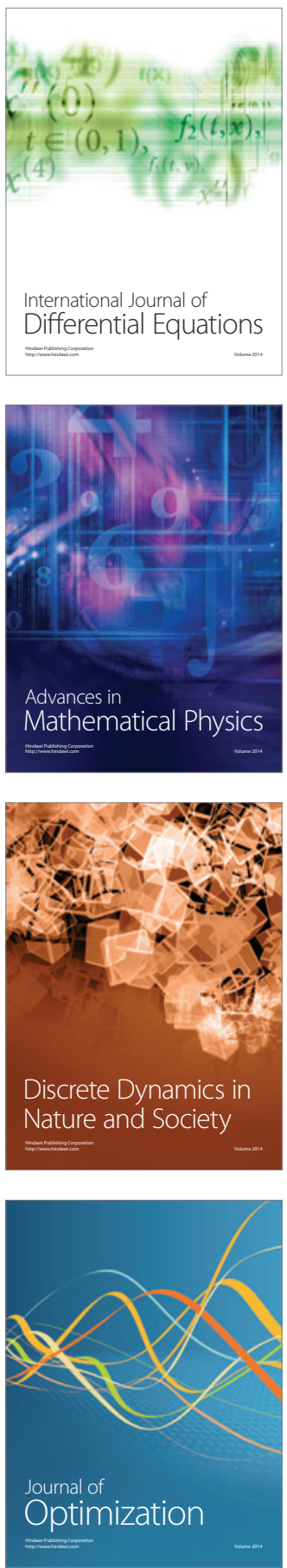